\title{
Exercise-therapy and education for individuals one year after anterior cruciate ligament reconstruction: a pilot randomised controlled trial
}

Brooke E. Patterson * (D), Christian J. Barton, Adam G. Culvenor, Randall L. Cooper and Kay M. Crossley

\begin{abstract}
Background: Guided rehabilitation beyond 6-months is rare following anterior cruciate ligament reconstruction $(A C L R)$, despite high prevalence of unacceptable symptoms and quality of life (QoL). Our primary aim was to determine the feasibility of a randomised controlled trial (RCT) evaluating a physiotherapist-guided intervention for individuals 1-year post-ACLR with persistent symptoms. Our secondary aim was to determine if a worthwhile treatment effect could be observed for the lower-limb focussed intervention (compared to the trunk-focussed intervention), for improvement in knee-related QoL, symptoms, and function.
\end{abstract}

Design: Participant- and assessor-blinded, pilot feasibility RCT.

Methods: Participant eligibility criteria: i) $12-15$ months post-ACLR; ii) $<87.5 / 100$ on the Knee injury and Osteoarthritis Outcome Score (KOOS) QoL subscale; and either a one-leg rise test $<22$ repetitions, single-hop $<90 \%$ limb symmetry; or Anterior Knee Pain Scale <87/100. Participants were randomised to lower-limb or trunk-focussed focussed exercise and education. Both interventions involved 8 face-to-face physiotherapy sessions over 16-weeks. Feasibility was assessed by eligibility rate ( $>1$ in 3 screened), recruitment rate ( $>4$ participants/month), retention ( $<20 \%$ drop-out), physiotherapy attendance and unsupervised exercise adherence $(>80 \%)$. Between-group differences for knee-related QoL (KOOS-QoL, ACL-QoL), symptoms (KOOS-Pain, KOOS-Symptoms), and function (KOOS-Sport, functional performance tests) were used to verify that the worthwhile effect (greater than the minimal detectable change for each measure) was contained within the $95 \%$ confidence interval.

Results: $47 \%$ of those screened were eligible, and 27 participants (3 participants/month; $48 \%$ men, $34 \pm 12$ years) were randomised. Two did not commence treatment, and two were lost to follow-up (16\% drop-out). Physiotherapy attendance was $>80 \%$ for both groups but reported adherence to unsupervised exercise was low $(<55 \%)$. Both interventions had potentially worthwhile effects for KOOS-QoL and ACL-QoL, while the lower-limb focussed intervention had potentially greater effects for KOOS-Sport, KOOS-Pain, and functional performance.

* Correspondence: B.Patterson@latrobe.edu.au

La Trobe Sport \& Exercise Medicine Research Centre, School of Allied Health,

Humans Services and Sport, La Trobe University, Bundoora 3086, Australia

C C The Author(s). 2021 Open Access This article is licensed under a Creative Commons Attribution 4.0 International License, which permits use, sharing, adaptation, distribution and reproduction in any medium or format, as long as you give appropriate credit to the original author(s) and the source, provide a link to the Creative Commons licence, and indicate if changes were made. The images or other third party material in this article are included in the article's Creative Commons licence, unless indicated otherwise in a credit line to the material. If material is not included in the article's Creative Commons licence and your intended use is not permitted by statutory regulation or exceeds the permitted use, you will need to obtain permission directly from the copyright holder. To view a copy of this licence, visit http://creativecommons.org/licenses/by/4.0/ The Creative Commons Public Domain Dedication waiver (http://creativecommons.org/publicdomain/zero/1.0/) applies to the data made available in this article, unless otherwise stated in a credit line to the data. 
(Continued from previous page)

Conclusions: A larger-scale RCT is warranted. All feasibility criteria were met, or reasonable recommendations could be made to achieve the criteria in future trials. Strategies to increase recruitment rate and exercise adherence are required. The potential worthwhile effects for knee-related QoL, symptoms, and function indicates a fully-powered RCT may detect a clinically meaningful effect.

Trial registration: Prospectively registered (ACTRN12616000564459).

Keywords: Anterior cruciate ligament, Rehabilitation, Physiotherapy

\section{Background}

Following anterior cruciate ligament reconstruction (ACLR), clinical practice guidelines recommend postoperative rehabilitation to continue for at least 9 to 12 months, or until achievement of sport-specific strength, functional and psychological criteria [72]. Yet, many patients have symptoms, muscle weakness and functional deficits that persist beyond 1-year post-ACLR [13, 55, $66,79]$, which may increase the risk of re-injury, symptomatic post-traumatic osteoarthritis (OA), and worse knee-related quality of life (QoL) [17, 18, 25, 26, 33, 53]. Yet, there are no clinical trials to suggest exercise and education beyond the typical rehabilitation period is feasible or beneficial for those who have not achieved an acceptable outcome within the first post-operative year.

Approximately $50 \%$ of individuals report unacceptable knee symptoms and QoL 1 to 2 years after ACLR [39, 55]. Minimal improvement occurs beyond 1 to 2 years $[55,65]$, and symptoms and QoL remain worse than their uninjured peers in the longer-term (> 5 years) [27, 55]. Persistent symptoms at 1-year post-ACLR often coexist with impairments in physical strength and function, and loss of knee confidence [16, 35]. Strength and functional performance impairments are typically defined as a difference in performance greater than 10\% between the ACLR and contralateral limb. Persistent symptoms and functional deficits at 1-year post-ACLR increase the risk of developing short-term $(<5$ years) and longer-term (5 to 10 years) symptoms, impaired knee-related QoL and OA $[15,25,53]$. Therefore, the one-year postoperative milestone provides an ideal window to identify "at risk individuals" with persistent symptoms, who have ceased supervised rehabilitation and for interventions to be implemented. Physiotherapist-guided exercisetherapy and education to address persistent physical impairments and symptoms, may be important to the secondary prevention of re-injury, post-traumatic OA, and poor QoL in young adults post-ACLR $[12,76]$.

The primary aim of this pilot study was to determine the feasibility of a randomised controlled trial (RCT) evaluating a physiotherapist-guided exercise-therapy intervention for individuals with persistent symptoms 1year post-ACLR. Our secondary aim was to determine if a worthwhile treatment effect was observed for the lower-limb focussed intervention (compared to the trunk-focussed intervention), for improvement in kneerelated QoL, symptoms, and function.

\section{Methods \\ Study design}

This double-blind (assessor and participant), parallelarm, pilot feasibility RCT was conducted in accordance with the National Health and Medical Research Council ethical guidelines [51], and reporting adheres to the Consolidated Standards of Reporting Trials (CONSORT) statement for pilot and feasibility studies [23] (Additional file 1). Ethical approval was gained from the La Trobe University Human Ethics Committee (HEC 16077). The trial was prospectively registered through the Australia and New Zealand Clinical Trials Registry (ACTRN12616000564459).

\section{Setting}

All assessments and treatments were conducted at two private physiotherapy clinics in Australia, located in Hobart or Melbourne.

\section{Participant recruitment and eligibility}

Individuals who had undergone a hamstring-tendon autograft ACLR 12 to 15 months prior were recruited from five surgical lists, advertisements at La Trobe University, or via social media (December 2016 to August 2017). Individuals aged 18 to 50 years who were 12 to 15 months post-ACLR were considered eligible if they scored $<87.5 / 100$ on the Knee injury and Osteoarthritis Outcome Score (KOOS) QoL subscale (threshold below which has been defined a symptomatic knee [24]), and met one of the following criteria; a) $<22$ repetitions on the one-leg rise test; b) single-hop $<90 \%$ limb symmetry index (LSI); or c) $<87 / 100$ on the Anterior Knee Pain Scale (AKPS) [44]. These functional performance thresholds can be associated with worse symptoms and poorer knee-related QoL in the proceeding years [15, 25, 61], and the AKPS threshold can be associated with worse functional performance at 1-year post-ACLR [16]. Exclusion criteria were: i) > 5 years between injury and ACLR; 
ii) subsequent injury (for which medical treatment was sought) or follow-up surgery to the ACLR knee; iii) another musculoskeletal, neurological, or cardiorespiratory condition influencing daily function; iv) unable to speak or read English; and v) unable to attend eight supervised sessions.

\section{Deviations from initial trial protocol}

Participants were initially deemed ineligible if they had sustained a previous ACL or knee injury to either limb prior to their recent ACLR. After commencing recruitment, it was evident that a previous knee injury was common in those with persistent symptoms, and these individuals have an increased risk of symptomatic posttraumatic OA [78]. The inclusion criteria were adjusted at the start of recruitment to include those with a previous ACL or knee injury. The single-hop performance LSI cut-off for eligibility was changed from 88 to $90 \%$, as recent evidence suggests $90 \%$ is the most common criterion used for return-to-sport clearance [9]. Hypothesis testing in a regression model was not performed as initially planned, due to the limitations of significance testing in clinical research [36], and this was not considered appropriate for a feasibility trial. Instead, the betweengroup differences and 95\% confidence intervals (CI) were used to verify that a worthwhile effect was contained within the CI [5]. We defined a potential worthwhile effect as greater than the minimal detectable change (MDC) score for the respective outcome measures where available. While the primary purpose of feasibility was implied throughout the trial registration and included as such in the trial title, we did not list feasibility as a separate outcome in the trial registration. We have maintained our focus on feasibility by including it as the primary aim of this pilot study. Several other exploratory PROs were outlined in the trial registration but were beyond the scope of this evaluation due to the primary aim of feasibility.

\section{Procedures}

Eligible participants underwent a baseline assessment with a blinded assessor (BP) and were randomised into one of two intervention groups. The same blinded assessor completed all follow-up assessments unaware of group allocation. Participant age, sex, body mass index (BMI), injury history, ACLR rehabilitation (i.e. self-reported duration) and surgical details (i.e. self-reported graft type, meniscal procedures), and previous activity level were obtained at baseline. All patient-reported outcomes (PROs) were completed via an online portal (Promptus, DS PRIMA, Melbourne, Australia).

\section{Randomisation and blinding}

Non-stratified, permuted block randomisation (random blocks of 3 or 6) occurred at a 2:1 (lower-limb focussed: trunk-focussed) ratio. The randomisation sequence was computer-generated using Excel. The administrative staff at the participating physiotherapy clinic revealed the allocation using sequentially numbered, sealed opaque envelopes. The administrative staff were blinded to block size, and entered the group allocation to the participant's clinical record for the physiotherapist. Participants were blinded to group allocation, to ensure allocation did not influence adherence, other treatment use, or increase the risk of drop-out. The physiotherapists were unable to be blinded to the allocation but were encouraged to deliver both interventions with equal enthusiasm and assertion of exercise value.

\section{Treating physiotherapists and treatment fidelity}

Treating physiotherapists were experienced ( $\geq 5$ years treating musculoskeletal patients) in ACLR rehabilitation, and completed a 4-h training session (led by BP) related to delivering both interventions. A manual, outlining the exercise prescription and progressions, manual treatment algorithm, education material, and trial procedures (attendance sheet, clinical notes, adherence monitoring) was provided to each physiotherapist (Additional file 2). Prescribed exercises were entered via Physitrack $\odot$ smartphone application for participants to access via the participant-facing application PhysiApp@ (Physitrack Ltd., London, UK).

\section{Interventions}

Participants were randomised to a lower-limb focussed or trunk-focussed exercise-therapy intervention, which were both delivered in eight face-to-face 30-min physiotherapy sessions over 16-weeks. Both interventions are reported below according to the Template for Intervention Description and Replication (TIDieR) guidelines [38] and the Consensus on Exercise Reporting Template (CERT) [64] (Table 1).

\section{Lower-limb focussed exercise-therapy intervention}

The lower-limb focussed intervention included standardised (with individualised progression) lower-limb, functional and cardiovascular exercises, and individualised, ACL-specific education (Additional file 2). The protocol was informed by current evidence-based recommendations [72], and developed by the research team, two of whom regularly (weekly) treated patients after ACLR ( $\mathrm{CB}$ and $\mathrm{RC}$ ). The lower-limb focussed exercise-therapy program targeted typical strength and functional impairments [79], and altered movement patterns [79] during sport-specific tasks related to ACL injury mechanisms (i.e., landing, and cutting). The eight areas in the exercise-program were: 1) movement retraining (e.g. 
Table 1 Summary of intervention delivery and components for both groups

\begin{tabular}{|c|c|c|}
\hline What & Lower-limb focussed intervention & Trunk-focussed intervention \\
\hline Who provides & \multicolumn{2}{|c|}{ Physiotherapists who have all undergone study-specific training } \\
\hline How & \multicolumn{2}{|c|}{ 1-to-1 face-to-face sessions to assess and progress unsupervised exercise-therapy program } \\
\hline Where & \multicolumn{2}{|c|}{$\begin{array}{l}\text { Physiotherapy sessions: Private clinics in Hobart and Melbourne } \\
\text { Unsupervised exercise-therapy program: Clinic/public gym, or home }\end{array}$} \\
\hline $\begin{array}{l}\text { When \& how } \\
\text { much }\end{array}$ & \multicolumn{2}{|c|}{$\begin{array}{c}\text { Physiotherapy 1-to-1 sessions: } 30 \text { min duration, weekly for } 4 \text { weeks then every } 2 \text { to } 3 \text { weeks for } 12 \text { weeks } \\
\text { Unsupervised exercise-therapy program: instructions provided via PhysiApp@, } 30 \text { to } 45 \text { min duration, minimum } 3 \text { sessions per week, } \\
\text { unsupervised }\end{array}$} \\
\hline \multirow[t]{2}{*}{ Tailoring } & $\begin{array}{l}\text { - Standardised lower-limb exercises (i.e. strength, power, balance), functional retraining } \\
\text { (e.g. plyometric, agility) and cardiovascular program } \\
\text { - Choice of priority exercises }{ }^{\mathrm{a}} \text { (from the standard set) was individualised } \\
\text { - Exercise progression was individualised } \\
\text { - Individualised education (e.g. exercise rationale, goal setting) } \\
\text { - Passive therapy treatment algorithm if appropriate (e.g. taping) }\end{array}$ & $\begin{array}{l}\text { - Standardised, non-specific trunk strength- } \\
\text { ening exercises } \\
\text { - Progression of exercises was individualised } \\
\text { - Optional stretching } \\
\text { - Standardised education (e.g. rationale for } \\
\text { trunk exercises) }\end{array}$ \\
\hline & \multicolumn{2}{|c|}{$\begin{array}{l}\text { Both groups: exercises progressed based on assessment of pre-defined criteria at each session (i.e., pain, swelling, technique) and resistance } \\
\text { training principles }\end{array}$} \\
\hline How well & \multicolumn{2}{|c|}{$\begin{array}{c}\text { Attendance at physiotherapy recorded by physiotherapists and clinic } \\
\text { Unsupervised exercise program adherence recorded by participants in PhysiApp@ } \odot \text { smartphone app or paper diaries, and monitored } \\
\text { by physiotherapists via Physitrack } \odot\end{array}$} \\
\hline
\end{tabular}

${ }^{a}$ Physiotherapists could choose 3 to 4 priority exercises (out of a possible 8), based on the participant's needs and goals. If necessary, all 8 exercise types were included, but it was not compulsory for all eight to be incorporated

landing); 2) lower-limb strength (e.g. squats); 3) balance (e.g. perturbation exercises); 4) hip-abductor strength; 5) calf strength; 6) trunk strength; 7) hip-extensor and knee-flexor strength; and 8) cardiovascular exercise (e.g. cycling, running, graded sport-specific activities). Each of the eight exercises had three or more phases of difficulty for individualised progression (Additional file 2). Physiotherapists were provided with a summary of the participant's injury history, goals, 3 to 4 priority exercises, and suggested starting phases based on baseline assessment. Physiotherapists could add target exercises based on participant need, but it was not compulsory for all eight exercises to be incorporated. Exercise progression was based on: i) good technique; ii) minimal irritability (i.e. $<2 / 10$ pain during/after and no swelling); iii) resistance training principles related to muscular strength and power [1]; and iv) participant-specific goals and feedback. Strength exercises were prescribed in 3 sets of 12 repetitions (each repetition performed as $2 \mathrm{~s}$ concentric, $1 \mathrm{~s}$ isometric, $2 \mathrm{~s}$ eccentric), and could be progressed to a power dosage prescribed in 3 to 5 sets of 5 to 10 repetitions $(<1 \mathrm{~s}$ concentric, 0 isometric, $2 \mathrm{~s}$ eccentric) [1]. Treating physiotherapists were encouraged to use the face-to-face sessions to check exercise technique, and adjust loads so that participants were reaching fatigue (i.e. they could not physically perform $>2$ more repetitions) after their prescribed dosage [1]. Thirty-minutes was considered an appropriate appointment duration to supervise at least 1 set of prescribed exercises (the other 2 sets could be completed unsupervised in the clinic gym), and provide education.

\section{Trunk-focussed exercise-therapy (control) intervention}

An active control intervention was chosen to ensure that both treatment groups received equal exposure to physiotherapy [37]. The trunk-focussed intervention was considered the active control, and included standardised (with individualised progression) trunk strengthening exercises, stretching and education. Physiotherapists could choose a minimum of three trunk strengthening exercises (from a maximum of five options), and each exercise had three or more phases of difficulty (Additional file 2). Exercises were prescribed according to resistance training principles; typically prescribed in 3 sets of $60 \mathrm{~s}$ (isometric), and progressed to achieve adequate fatigue (i.e., could not physically perform $>5$ more seconds) [1]. Lower-limb and trunk stretching appropriate to the participant, could be prescribed (Additional file 2). The trunk exercises were predominantly isometric, nonweight-bearing, and had minimal lower-limb involvement and thus, were not expected to impact kneerelated QoL, symptoms, or function. This was chosen as the control intervention as trunk muscle strength deficits has not been reported following ACLR, nor has addressing trunk strength been investigated to impact knee-related outcomes following ACLR. Trunk exercises were considered to provide a credible intervention to enhance control participant's blinding to group allocation and minimise drop-outs.

\section{Unsupervised exercise-therapy program (both groups)}

Participants in both groups were prescribed an unsupervised exercise-therapy program relevant to their 
allocation, to be completed 3 times per week, at home or in a gym, to optimise likelihood of muscular strength and power improvements [29]. Physiotherapists entered participant's exercises via the Physitrack $\odot$ app, for the participant to use PhysiApp® to guide exercises and record adherence on their own smartphone, tablet or computer. Paper diaries of the exercise-therapy programs were provided as required. PhysiApp $\odot$ included video examples (created specifically for the trial) of correct (and incorrect) technique for each exercise (Additional file 3), and exercise dosage (e.g. number of sets/ repetitions, time under tension, external load, rest time) according to resistance training and muscle adaptation guidelines $[1,69]$. Co-interventions were discouraged but if participants chose to receive other treatment, they recorded them on an "other treatments calendar". The trunk-focussed unsupervised program could be completed at home with minimal equipment. When gym equipment was required for the lower-limb focussed unsupervised program, gym access was provided free of charge.

\section{Education component (both groups)}

Both groups received education, including face-to-face discussion and/or provision of handouts (Additional file 3). Handouts for the lower-limb focussed group covered the following topics: i) surgical information and postoperative expectations; ii) goal setting and return-tosport criteria; iii) injury prevention; iv) psychosocial influences on recovery; and v) post-traumatic OA risk. The purpose of the education for the lower-limb focussed group was to provide informational support regarding ACL-specific topics and address common knowledge gaps regarding evidence-based rehabilitation [6], and psychosocial support for kinesiophobia, fear of re-injury, confidence, or negative lifestyle modifications (e.g. weight gain) known to influence outcomes [11, 56, 70]. For the trunk-focussed group, physiotherapists could deliver standardised education on the rationale for trunk strengthening (e.g. theoretical influence of lumbopelvic stability on lower-limb biomechanics), or provide handouts/ face-to-face discussion on the topics "surgical information and post-operative expectations", "psychosocial influences on recovery", and "post-traumatic OA risk" (Additional file 3).

\section{Primary outcome: feasibility}

Feasibility was assessed according to previously published recommendations [47]. Proceeding to a full-scale RCT was deemed feasible if all criteria were met, or reasonable amendments could be made to achieve these criteria in future trials [3].

Recruitment, adherence and retention was evaluated by: i) recruitment rate (criterion: 4 participants per month); ii) proportion of eligible participants who were willing to enrol (criterion: $>80 \%$ ); iii) physiotherapy attendance rate (criterion: $>80 \%$ ); iv) adherence to unsupervised exercise-therapy program (criterion: $>80 \%$ ); and v) proportion of drop-outs (criterion: $<20 \%$ ).

Acceptability of the study protocol was assessed via the appropriateness of the inclusion criteria (criterion: at least 1 in 3 eligible), and acceptability of the intervention content, delivery, adherence monitoring, and barriers or facilitators to adherence. Acceptability was determined via informal interviews conducted with participants and physiotherapists (Additional file 4).

Adverse events (i.e., any injury or illness requiring medical attention as a result of participating in the trial)) were noted by the physiotherapist on a standardised recording sheet (criterion: $<10 \%$ of all participants). Painlevel (on a visual analogue scale; $0=$ no pain, $10=$ worst possible pain) during the unsupervised exercise-therapy program was entered on PhysiApp@ $\odot$ by participants (criterion: each participant mean $<2 / 10$ across all sessions).

Randomisation integrity was determined by contamination between groups (reported by participant or physiotherapist) (criterion: 0\% contamination), or knowledge of group allocation by the participants or assessor (criterion: < $10 \%$ unblinded).

Acceptability of the outcome measures was determined by the time needed to collect the data, and completeness of the outcome measures at baseline and follow-up (criterion: > 90\%).

\section{Secondary outcomes \\ Patient-reported outcomes}

Knee-related QoL was assessed via the KOOS-QoL and ACL-QoL. The KOOS-QoL is one of the five KOOS subscales, and evaluates knee-related QoL [63]. The KOOS-QoL has the highest content validity of all subscales and the greatest responsiveness in young adults following knee injury [10]. The ACL-QoL was designed to assess additional domains (e.g. work-related, social and emotional) of knee-related QoL specific to a young, active ACL-injured population [49]. The KOOS-QoL and ACL-QoL are converted to a total score out of 100 $(0=$ extreme problems, $100=$ no problems $)$. The KOOSQoL and ACL-QoL have established content validity (Cronbach's alpha >0.76), test-retest reliability (ICC> 0.86 ), and responsiveness (effect sizes >0.5) [10, 46]. The MDC is $8-10$ points for KOOS-QoL [63], and 12points for ACL-QoL [46].

The KOOS subscales of pain, symptoms, and sport were assessed, and all combined with the KOOS-QoL, to calculate an overall $\mathrm{KOOS}_{4}$ score. The KOOS individual subscales are valid, reliable and responsive following ACL injury [10]. Psychological readiness for return-tosport (a common goal of ACLR), and fear of re-injury 
was measured by the ACL Return to Sport Index (ACLRSI) [74]. The ACL-RSI has established test-retest reliability $(\mathrm{ICC}=0.89)$ and responsiveness $(\mathrm{MDC}=19$ points) [45], and validity with higher scores associated with better return-to-sport rates, self-reported symptoms and function [45, 75]. The global rating of change (GROC) on a 7-point Likert scale ("much worse" to "much better") measured separately for knee pain and knee function; and the change in proportion of patients answering "yes" to the patient acceptable symptom state (PASS) question [39] were evaluated. The GROC has good face validity (Pearson's $\mathrm{r}=0.72$ to 0.90 ), test-retest reliability (ICC > 0.90), responsiveness following knee injury (0.5 to 2.7 points on 7 -point scale), and construct validity (e.g. correlated with changes in hop tests) [40]. The PASS assists in interpretation of improvement in PROs by evaluating the concept of "feeling good" as opposed to "feeling better" [71], and answering yes to "PASS" corresponds with better KOOS scores after ACL injury [39].

\section{Functional performance outcomes}

Functional performance outcomes were measured at baseline and follow-up, including the single-hop (maximum distance on one hop forward) [34], side-hop (maximum number of hops over two parallel lines $40 \mathrm{~cm}$ apart in $30 \mathrm{~s}$ ) [34], and one-leg rise test (maximum number repetitions from a standardised height) [68]. We recorded the raw score (e.g. cm hopped) on the ACLR and contralateral limb, and calculated the LSI (score of ACLR knee divided by contralateral knee, multiplied by 100, expressed a percentage). The hop-tests and one-leg rise have high intra-rater reliability $(\mathrm{ICC}>0.80)$ and responsiveness after knee injury [7, 34, 60].

\section{Data analysis}

The sample size of 27 was not formally determined. It was based on previous pilot RCTs evaluating healthprofessional guided interventions for musculoskeletal conditions [41, 67], and deemed sufficient to assess the feasibility criteria. Participants who completed baseline and follow-up evaluations were included in the analysis, as recommended in the CONSORT guidelines [48]. Feasibility outcomes were reported descriptively. The majority $(>50 \%)$ of baseline and follow-up scores, and the change scores for the patient-reported and functional performance outcomes were normally distributed (assessed with Shapiro-Wilk's test). Therefore, withingroup, and between-group differences were reported as mean $\pm \mathrm{SD}$, and mean and $95 \%$ confidence interval $(\mathrm{CI})$, respectively. The treatment effect for the respective outcome measures was considered potentially worthwhile if the MDC was contained within the $95 \% \mathrm{CI}$ of the mean between-group difference [5]. Activity level, GROC, and PASS outcomes were reported descriptively. Decision criteria for progression to a full-scale RCT was based on: i) all feasibility criteria being met, or reasonable recommendations to achieve criteria in future trials and ii) presence of a potentially worthwhile treatment effect for knee-related QoL, symptoms, and function.

\section{Results \\ Feasibility}

All feasibility criteria were met, or reasonable recommendations could be made to achieve the criteria in future trials (Table 2). Eighty people expressed interest in participation via response to letters from their surgeon $(n=55)$, or advertisements on social media and La Trobe University $(n=25)$ over a 9 -month period. $72 \%(n=57)$ agreed to be screened, with $47 \%$ of those screened ( $n=$ 27) deemed eligible (Fig. 1). The results of each aspect of feasibility are summarised in Table 2, with the detailed feedback provided by participants at follow-up provided in Additional file 4.

\section{Participant characteristics}

The trunk-focussed group had a higher proportion of men, a higher proportion participating in Level 1 or 2 sports pre-injury, and higher proportion of concomitant meniscal surgery at the time of ACLR (Table 3).

\section{Patient-reported outcomes}

The desired treatment effect for KOOS-QoL (improvement $>8-10$ points) and ACL-QoL (improvement >12 points) was contained within the 95\% CI (Table 4). Additional file 5 reports the individual treatment responses for KOOS-QoL and ACL-QoL, and proportion with improvements greater than the MDC. The KOOS-Pain, KOOS-Sport, $\mathrm{KOOS}_{4}$, GROC and PASS indicated both groups improved, potentially to greater extent in the lower-limb focussed group (Table 4). The MDC was contained within the 95\% $\mathrm{CI}$ for KOOS-Pain, KOOS-Sport and $\mathrm{KOOS}_{4}$ (Table 4). Majority (87\%) were at least "better" for knee function and knee pain in the lower-limb focussed group, compared to 50 and $75 \%$ respectively in the trunk-focussed group. Satisfaction (PASS question) with current knee function improved in the lowerlimb focussed group from 27 to 67\%, but remained the same in the trunk-focussed group (63\%).

\section{Functional performance outcomes}

The MDC (where available) was contained within the 95\% CI for all functional performance tests, except for the side-hop ACLR limb performance (Table 4). Additional file 5 demonstrates the proportion of participants who have improvements greater than the MDC, and highlights the large individual variation in change in functional performance in both groups. 
Table 2 Feasibility outcomes

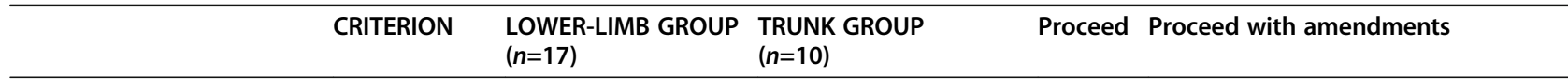

\section{Recruitment, retention, adherence}

$\begin{array}{lllll}\begin{array}{l}\text { Recruitment rate } \\ \text { Enrolment rate }\end{array} & >4 \text { per month } & 3 \text { participants per month } \\ \text { Drop-out rate } & <20 \% & \begin{array}{l}100 \% \text { completed baseline assessment, were } \\ \text { enrolled and randomised }\end{array} \\ \begin{array}{llll}\mathrm{n}=2(12 \%)^{\mathrm{a}} & \mathrm{n}=2(20 \%)^{\mathrm{b}}\end{array} \\ \begin{array}{l}\text { Exysiotherapy attendance } \\ \text { Exercise adherence }\end{array} & >80 \% & \begin{array}{l}\text { Mean }=89 \% \text { of } \\ \text { intended 8 sessions }\end{array} & \begin{array}{l}\text { Mean }=86 \% \text { of } \\ \text { intended 8 sessions }\end{array} \\ & >80 \% & \begin{array}{l}52 \% \text { of sessions } \\ \text { completed }\end{array} & \begin{array}{l}48 \% \text { of sessions } \\ \text { completed }\end{array}\end{array}$

Strategies to increase recruitment rate

Yes

Yes

Yes

No

completed completed

\section{Study protocol acceptability}

\section{Eligibility rate \\ 1 in 3 \\ Acceptability of intervention Descriptive \\ to physiotherapists \\ Acceptability of intervention Descriptive to participants}

Barriers to adherence Descriptive

\section{Adverse Events}

$\begin{array}{ll}\text { Injury or illness } & <20 \% \\ \text { Pain during/after exercise } & <2 / 10\end{array}$

\section{Randomisation integrity}

Integrity of blinding

$90 \%$

Group contamination

$0 \%$

Nil
$47 \%$ of interested participants were eligible

- Training and supportive material sufficient - Reflected clinical practice, but time allocation insufficient

- Appointment duration/frequency, and facilities appropriate.

- Interventions were credible and acceptable

Work, study and family commitments, lack of motivation, boredom with exercises

$n=4(24 \%)$ unrelated to intervention ${ }^{c}$

Mean pain <2/10 for each participant (across all sessions)

Assessor unblinded for $n=1$

1 participant (medical professional) knew they were in the "control" group

Physiotherapists reported often discussing patientspecific topics

\section{Acceptability of outcomes}

$\begin{array}{ll}\text { Time to collect data } & <90 \mathrm{~min} \\ \text { Completeness of PROs } & >90 \% \\ \text { Completeness of functional } & >90 \% \\ \text { performance outcomes } & \end{array}$

Adherence monitoring Descriptive
Baseline and follow-up assessments were completed in 60-90 min

All 23 participants who finished the trial completed PROs, with no missing data

$\begin{array}{ll}16 \%(n=4) \text { did not } & \begin{array}{ll}10 \%(n=1) \text { did not } \\ \text { complete follow-up }\end{array} \\ & \begin{array}{l}\text { complete follow-up } \\ \text { (located internationally) }\end{array}\end{array}$

- 23 used PhysiApp@ and 2 used paper diaries - Enjoyed the accountability and motivation PhysiApp@ provided, and the videos for exercise technique

- Inconsistently used PhysiApp@ to record adherence (i.e. technical issues, or forgot to use the app as they knew their program)
Yes

Appointments $>30$ min or provide additional more frequent appointments

Yes

Control education difficult to standardise for control groups
Yes

Yes

No

No
Consider PROs as primary outcome for complete data

Strategies to increase adherence to data entry (e.g. incentives, interactive features such as benchmarking, education)

\footnotetext{
${ }^{a} n=1$ severe increase in knee pain, $n=1$ unable to commit to requirements

${ }^{\mathrm{b}} n=2$ decided they could not commit to the trial before commencing interventions

${ }^{c} n=1$ severe increase in knee pain (group fitness class); $n=2$ hamstring strains (sprint training, sprint in basketball match); $\mathrm{n}=1$ ankle sprain (football training)

${ }^{d}$ Consider high-speed running and sport-specific programs to reduce risk of other soft tissue injuries

e $n=2$ could not complete hop-test (recovering from ankle sprain and hamstring strain), $\mathrm{n}=2$ could not attend (located internationally, work commitments).
} 


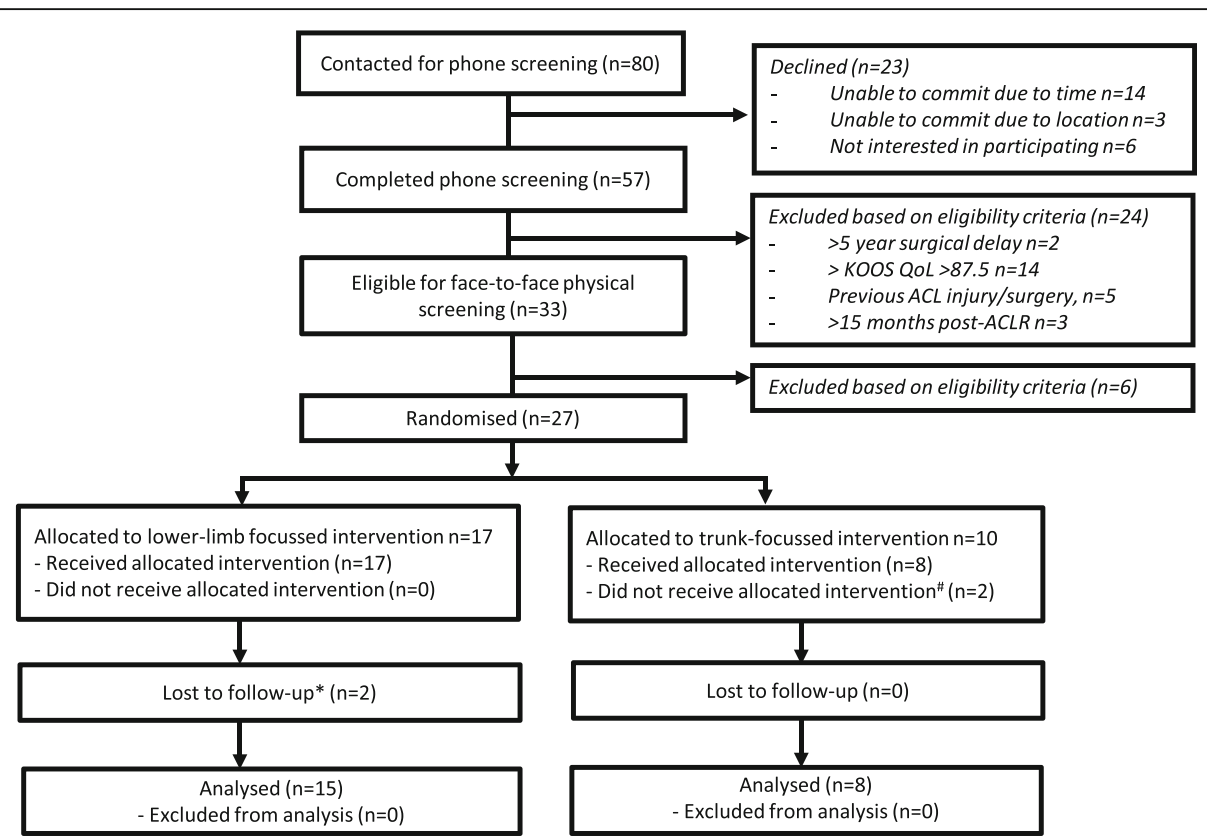

Fig. 1 Flow of participants through the study. KOOS-QoL=Knee injury and Osteoarthritis Outcome Score Quality of Life subscale; ACL=anterior cruciate ligament; $\mathrm{ACLR}=$ anterior cruciate ligament reconstruction. ${ }^{*} n=2$ unable to find appointments to suit work/study commitments. ${ }^{*} n=1$ severe increase in knee pain, $n=1$ unable to commit to requirements

\section{Discussion}

The results of this study suggest it is worthwhile proceeding to a large-scale RCT evaluating the effectiveness of a physiotherapist-guided lower-limb focussed exercise-therapy and education intervention for young adults who have persistent symptoms 1 -year post-ACLR. All feasibility criteria were either met, or reasonable recommendations could be made to achieve the criteria in future trials. Additionally, worthwhile treatment effects were observed in participants receiving the lower-limb focused intervention for knee-related symptoms, function and QoL.
Feasibility: recruitment, retention, attendance and protocol acceptability

Of those screened, almost half (47\%) were eligible, and we achieved a modest recruitment rate (3 per month). For a large-scale RCT, the number of participating surgeons (and study advertising) would need to be increased, which is possible due to the large number of ACLRs performed each year [50]. Although all eligible participants were willing to enrol, two participants did not commence the intervention, and two others dropped out during the intervention, resulting in an overall dropout rate of $16 \%$, which is considered acceptable [28].

Table 3 Participant characteristics at baseline

\begin{tabular}{|c|c|c|}
\hline & Lower-limb focussed group $(n=17)$ & Trunk-focussed group $(n=10)$ \\
\hline Age, mean $\pm S D$ years & $34 \pm 12$ & $33 \pm 12$ \\
\hline Sex, no. (\% male) & $6(35 \%)$ & $7(70 \%)$ \\
\hline Body mass index, mean $\pm \mathrm{SD} \mathrm{kg} / \mathrm{m}^{2}$ & $24.7 \pm 2.8$ & $25.4 \pm 3.8$ \\
\hline \multicolumn{3}{|l|}{ Pre-injury activity level ${ }^{a}$, no. (\%) } \\
\hline Level 1 or 2 & $19(53 \%)$ & $10(100 \%)$ \\
\hline Level 3 & $6(35 \%)$ & $0(0 \%)$ \\
\hline Level 4 & $2(12 \%)$ & $0(0 \%)$ \\
\hline Concomitant meniscal procedure ${ }^{\mathrm{b}}$, no. (\%) & $9(53 \%)$ & $3(30 \%)$ \\
\hline Ceased supervised PT, median (IQR) months & $7(6)$ & $6(5)$ \\
\hline
\end{tabular}

SD Standard deviation, PT Physiotherapy

${ }^{a}$ According to Grindem classification system [32]. Level 1=pivoting/jumping/hard cutting sports (e.g. football), Level 2=pivoting/jumping sports but less intense cutting (e.g. volleyball), Level 3 sports=straight line activities (e.g. running, weight-lifting), and Level $4=$ sedentary

${ }^{b}$ at the time of ACLR, reported by participants at baseline assessment 
Table 4 Secondary outcomes at baseline and follow-up

\begin{tabular}{|c|c|c|c|c|c|c|c|c|}
\hline & \multicolumn{3}{|c|}{$\begin{array}{l}\text { Lower-limb focussed } \\
\text { intervention }(n=15)\end{array}$} & \multicolumn{3}{|c|}{$\begin{array}{l}\text { Trunk-focussed } \\
\text { intervention }(n=8)\end{array}$} & \multirow{2}{*}{$\begin{array}{l}\text { Lower-limb vs trunk } \\
\text { mean difference in } \\
\text { change }{ }^{*}(95 \% \mathrm{Cl})\end{array}$} & \multirow[t]{2}{*}{ Previously published MDC values } \\
\hline & Baseline & $\begin{array}{l}\text { Follow- } \\
\text { up^ }\end{array}$ & $\overline{\text { Change }}$ & Baseline & $\begin{array}{l}\text { Follow- } \\
\text { up^ }\end{array}$ & Change & & \\
\hline ACL-QoL & $45 \pm 20$ & $64 \pm 20$ & $20 \pm 17$ & $56 \pm 9$ & $78 \pm 16$ & $22 \pm 13$ & $-2.5(-18.2$ to 13.2$)$ & 12 points (Lafave et al., 2017) \\
\hline KOOS-QoL & $39 \pm 20$ & $62 \pm 23$ & $23 \pm 25$ & $52 \pm 14$ & $67 \pm 19$ & $16 \pm 12$ & $7.1(-12.3$ to 26.4$)$ & $\begin{array}{l}8 \text { to } 10 \text { points (Roos \& Lohmander, } \\
\text { 2003) }\end{array}$ \\
\hline $\begin{array}{l}\text { KOOS- } \\
\text { Symptoms }\end{array}$ & $68 \pm 23$ & $74 \pm 19$ & $7 \pm 17$ & $81 \pm 9$ & $90 \pm 9$ & $9 \pm 7$ & $-2.0(-15.7$ to 11.6$)$ & $\begin{array}{l}8 \text { to } 10 \text { points (Roos \& Lohmander, } \\
\text { 2003) }\end{array}$ \\
\hline KOOS-Pain & $77 \pm 15$ & $86 \pm 12$ & $9 \pm 14$ & $90 \pm 7$ & $92 \pm 8$ & $2 \pm 7$ & $6.7(-4.0$ to 17.9$)$ & $\begin{array}{l}8 \text { to } 10 \text { points (Roos \& Lohmander, } \\
\text { 2003) }\end{array}$ \\
\hline KOOS-Sport & $57 \pm 24$ & $77 \pm 22$ & $20 \pm 25$ & $76 \pm 1$ & $83 \pm 22$ & $8 \pm 13$ & 12.1 ( -7.9 to 32.0$)$ & $\begin{array}{l}8 \text { to } 10 \text { points (Roos \& Lohmander, } \\
\text { 2003) }\end{array}$ \\
\hline $\mathrm{KOOS}_{4}$ & $60 \pm 17$ & $75 \pm 17$ & $15 \pm 18$ & $75 \pm 9$ & $83 \pm 14$ & $9 \pm 7$ & $5.9(-7.9$ to 19.8$)$ & $\begin{array}{l}8 \text { to } 10 \text { points (Roos \& Lohmander, } \\
\text { 2003) }\end{array}$ \\
\hline ACL-RSI & $36 \pm 18$ & $53 \pm 22$ & $17 \pm 18$ & $41 \pm 18$ & $67 \pm 24$ & $26 \pm 22$ & $-9.2(-27.2$ to 8.7$)$ & 19 points (Kvist et al., 2013) \\
\hline \multicolumn{9}{|l|}{ Single-hop } \\
\hline $\mathrm{ACLR}(\mathrm{cm})$ & $65 \pm 42$ & $97 \pm 33$ & $33 \pm 34$ & $108 \pm 39$ & $115 \pm 42$ & $8 \pm 9$ & $24.1(-5.9$ to 54.1$)$ & $\begin{array}{l}14 \text { cm (Kockum \& Heijne, 2015; Reid } \\
\text { et al., 2007) }\end{array}$ \\
\hline $\begin{array}{l}\text { Contralateral } \\
(\mathrm{cm})\end{array}$ & $93 \pm 30$ & $106 \pm 32$ & $14 \pm 18$ & $116 \pm 25$ & $120 \pm 34$ & $4 \pm 15$ & $9.6(-8.9$ to 28.2$)$ & $\begin{array}{l}14 \mathrm{~cm} \text { (Kockum \& Heijne, 2015; Reid } \\
\text { et al., 2007) }\end{array}$ \\
\hline LSI (\%) & $59 \pm 38$ & $88 \pm 11$ & $29 \pm 37$ & $90 \pm 21$ & $94 \pm 12$ & $4 \pm 10$ & $15.5(-27.7$ to 58.7$)$ & $\begin{array}{l}\text { 8\% (Kockum \& Heijne, 2015; Reid } \\
\text { et al., 2007) }\end{array}$ \\
\hline \multicolumn{9}{|l|}{ Side-hop } \\
\hline ACLR (reps) & $8 \pm 8$ & $16 \pm 12$ & $9 \pm 9$ & $20 \pm 13$ & $29 \pm 19$ & $9 \pm 8$ & $-0.6(-9.4$ to 8.7$)$ & $\begin{array}{l}11 \text { reps (Kockum \& Heijne, 2015; } \\
\text { Reid et al., 2007) }\end{array}$ \\
\hline $\begin{array}{l}\text { Contralateral } \\
\text { (reps) }\end{array}$ & $9 \pm 9$ & $17 \pm 12$ & $8 \pm 10$ & $23 \pm 16$ & $31 \pm 21$ & $9 \pm 15$ & $-0.5(-13.4$ to 12.2$)$ & $\begin{array}{l}11 \text { reps (Kockum \& Heijne, 2015; } \\
\text { Reid et al., 2007) }\end{array}$ \\
\hline LSI $(\%)^{a}$ & $71 \pm 42$ & $82 \pm 29$ & $11 \pm 28$ & $73 \pm 13$ & $94 \pm 14$ & $21 \pm 18$ & $-10.4(-43.1$ to 22.2$)$ & $\begin{array}{l}\sim 10 \% \text { (Kockum \& Heijne, 2015; Reid } \\
\text { et al., 2007) }\end{array}$ \\
\hline \multicolumn{9}{|l|}{ One-leg rise } \\
\hline ACLR (reps) & $17 \pm 16$ & $33 \pm 15$ & $17 \pm 14$ & $27 \pm 19$ & $34 \pm 20$ & $7 \pm 11$ & $9.9(-4.1$ to 23.9$)$ & Not available \\
\hline $\begin{array}{l}\text { Contralateral } \\
\text { (reps) }\end{array}$ & $25 \pm 19$ & $35 \pm 15$ & $10 \pm 14$ & $31 \pm 15$ & $34 \pm 15$ & $4 \pm 7$ & $6.4(-6.5$ to 19.3$)$ & Not available \\
\hline LSI $(\%)^{b}$ & $67 \pm 57$ & $98 \pm 9$ & $31 \pm 54$ & $68 \pm 32$ & $83 \pm 47$ & $16 \pm 32$ & 15.7 ( -40 to 71.5$)$ & Not available \\
\hline
\end{tabular}

ACLR Anterior cruciate ligament reconstructed limb, ACL-RSI ACL Return to Sport Index, ACL-QoL Anterior Cruciate Ligament Quality of Life questionnaire, CI Confidence interval, KOOS Knee injury and Osteoarthritis Outcome Score, LSI Limb symmetry index, MDC Minimal detectable change, SD Standard deviation, QoL Quality of life

* Positive value indicates between-group differences are in favour of the lower-limb focussed group

$\wedge n=3$ participants did not complete functional performance follow-up (could not attend due to being overseas, or work commitments). An additional 2 participants did not complete the single-hop and side-hop tests as they were recovering from adverse events ( $n=1$ hamstring strain and $n=1$ ankle sprain). ${ }^{a} n=3$ not included for LSI calculation at baseline ( 3 in lower-limb focussed), and $n=3$ not included at follow-up ( 2 in lower-limb focussed, 1 in trunk-focussed), as unable to perform a valid score on either limb

${ }^{\mathrm{b}} n=5$ not included for $\mathrm{LSI}$ calculation at baseline ( $\mathrm{n}=3$ in lower-limb focussed group and $n=2$ in trunk-focussed group), and $n=1$ (in trunk-focussed group) not included at follow-up, as unable to perform a valid score on either limb $\sim$ Values are mean+/-SD unless otherwise indicated

Physiotherapy attendance was high (86 to $89 \%)$, which is similar to previous physiotherapist-guided exercisetherapy RCTs (> 80\%) for lower-limb musculoskeletal conditions in young adults [2, 41]. Suggestions during feedback from drop-outs and those who attended less than $80 \%$ of study appointments $(n=5)$ aligns with previous reported strategies to maintain attendance - i.e. increasing appointment availability after hours, exercise variety, and strategies to increase motivation [58]. These strategies, in addition to consideration of telehealth appointments, and multiple clinic locations might reduce drop-outs and improve attendance in future trials. Longer appointment duration or more frequent appointments may be required in future trials to provide physiotherapists with sufficient time to review exercise programs and provide education. Those with persistent symptoms may also require additional informational and emotional support, considering their knee-related QoL, 
symptoms, and function is considerably lower than most patients 1-year post-ACLR [55, 65].

\section{Feasibility: adherence to the unsupervised exercise- therapy program}

According to Physitrack $\odot$ adherence data, only half of the prescribed unsupervised exercise-therapy program sessions were completed. However, these data are likely to under-estimate true exercise adherence in this trial, as participants reported inconsistently entering their adherence data in Physiapp@ $\odot$ due to technical difficulties, and rarely using the app once familiar with the exercises. Regardless of true adherence rates, participants did report typical barriers to exercise adherence, including other commitments (work, study and family), and reduced motivation [30, 73]. Exercise adherence rates were lower than previous reports for rehabilitation during the first 6-months following ACLR (75 to 80\%) [8, 59]. This may reflect the burden of exercise-therapy on participants who have already endured unsuccessful rehabilitation with the physical, mental and time commitment it entails. Strategies to increase adherence (to the unsupervised exercise-therapy program and monitoring system) may include goal setting [77], incentivisation, supervised group classes, or alternate exercise options (e.g. nongym based) [30]. Personalised adherence monitoring data collection methods, including paper diaries, email or text questionnaires, and strategies to maintain engagment with apps (e.g. positive reinforcement, benchmarking) should be considered.

\section{Feasibility: adverse events, integrity of group allocation, acceptability of outcomes}

Two participants sustained hamstring strains in their ACLR limb, and one sustained an ankle sprain as they returned to sporting activities. Graded return to highspeed running protocols should be emphasised in future trials to reduce soft tissue injury risk, especially given ACL injury is a well-recognised risk factor for hamstring strain [31]. Future trials should include strategies to maintain participant blinding (e.g. control interventions that are credible), and sufficient study personnel to maintain at blinding of assessors. Completion of followup PROs (100\%) and functional performance measures (81\%), suggests PROs may be the most appropriate outcome tool to optimise completion in future trials.

\section{Future recommendations: treatment effects for knee- related QoL}

A worthwhile effect ( $>\mathrm{MDC}$ ) was observed for both the lower-limb and trunk-focussed interventions for the KOOS-QoL and ACL-QoL. Our results are consistent with other trials which comparing two types of physiotherapist-guided exercise interventions [2, 43].
The trunk-focussed group was hypothesised to have minimal effect on knee-related QoL, but greater trunk strength and endurance may improve perceived performance in sport and work-related activities, resulting in better QoL. Indeed, the trunk-focussed group had improvements in self-reported function (KOOS-Sport) which equalled the MDC (8 points). Improvements in knee-related QoL may be more strongly influenced by education (provided in both groups). In both groups, physiotherapists were able to educate participants and address psychological factors (e.g. kinesiophobia, fear, confidence), which are known determinants of adherence, recovery and self-reported outcomes after sportsrelated knee injury [30, 70]. The physiotherapists reported discussing patient and ACL-specific topics with the trunk-focussed participants, although directed not to do so in the study protocol, which may have had a direct or indirect effect on knee-related QoL. Future RCTs may consider evaluating the effects exercise-therapy and education with a comparator that better reflects usual care (e.g. self-directed exercise), and/or including a waitlist control. Given health-professional delivered education alone may be effective in young people with persistent knee pain [22], future trial designs might compare: (i) exercise-therapy versus education alone; and/or (ii) exercise-therapy with and without education, to guide interventions for those at risk of post-traumatic OA after ACLR.

\section{Future trial recommendations: treatment effects for knee- related function}

While the study was not powered to detect betweengroup differences, the lower-limb focussed group had greater improvements in KOOS-Sport, single-hop and one-leg rise likely to be clinically meaningful in a larger trial. However, these findings should be interpreted with caution due to the wide CIs, and between-groups differences at baseline particularly for the ACLR limb functional performance. In the lower-limb focussed group, the LSI improvements for the single-hop (29\%) were larger than those in the ACL-SPORTS trial (10\%) with a similar lower-limb focussed intervention [2]. This larger improvement we observed may be due the lower baseline function of our participants compared to the ACLSPORTS trial where all participants had already achieved $\geq 80 \%$ LSI, begun running, and had no pain [2]. Future studies should also consider that LSI improvement can reflect worsening contralateral limb function, rather than improved ACLR limb function [54]. Therefore, it is important to note that in the current interventional study, LSI improvements occurred alongside clinically meaningful improvements in both limbs, indicating the increase in LSI was due to greater improvement in the ACLR limb. Given poor function on hop-tests at 1-year 
post-ACLR may be associated with an increased risk of future OA [53, 57] and re-injury [33], addressing persisent functional deficits may be an important step forward in secondary prevention of post-traumatic knee $\mathrm{OA}$. Considering the influence of the lower-limb focussed intervention in this study on OA risk factors, future larger-scale trials should consider longer-term follow-up and include imaging assessment to determine structural joint trajectory and relationship with symptoms [19], physical activity monitoring, healthcare utilisation, and cost-effectiveness evaluation.

\section{Recommendations for future trials: intervention content and format}

Despite improvements, knee-related function and QoL remained lower than uninjured normative values $[4,42$, 52], and satisfaction with knee function was less than $70 \%$ in the lower-limb focussed group at follow-up, indicating that the lower-limb focussed intervention could be improved for future trials. Future trials may consider a longer intervention, with more frequent supervised sessions (either 1-to-1 or group exercise classes) to provide further opportunity for education and exercise progression to address persistent impairments. Some lowerfunctioning patients with persistent symptoms at 1-year post-ACLR may not wish to return-to-sport and have reduced motivation to complete plyometric and agility exercises. Future trials should consider a pragmatic individualised approach to exercise prescription, allowing the physiotherapist to choose from a set of exercises according to the patient's needs and goals. Isolated quadriceps exercises (e.g. knee extension) may be important to include in future interventions for those with persistent symptoms, due to the known associations between quadriceps weakness and development of symptomatic OA in the general population [20].

\section{Limitations and recommendations for full-scale RCT}

Given this was a pilot feasibility study, it did not have adequate power to establish superiority of one intervention over the other. Further, the lower-limb focussed group started with worse knee-related QoL and function, compared to the trunk-focussed group (Table 4), allowing greater room for improvement compared to the trunk-focussed group. A larger sample size would likely reduce baseline between-group heterogeneity. Future large-scale RCTs, including stratification for factors that may affect baseline status or treatment response (e.g. sex) [21] are now needed. Many participants (> 50\%) had a surgical review during the trial, and were given "clearance for return-to-sport", which may have improved PROs in both groups. Future trials should regularly (weekly or monthly) monitor all types of physical activity completed during the intervention period. Despite these limitations, this was a pilot feasibility study, with the purpose of recognizing improvements that could be made to the study design and protocols for future trials. Consistent with other ACLR cohorts [62] and RCTs [2], there was large individual variation in both groups for baseline scores (i.e., SDs) and changes between baseline and follow-up for all primary outcomes (Additional file 5). We did not assess lower-limb or trunk strength so we cannot indicate if the improvements in functional performance or PROs were mediated by strength increases. Future trials should include muscle capacity (strength, power) testing, to also ensure that adequate loading and progression has occurred to stimulate muscle capacity improvements [14]. Participating surgeons and clinic locations were limited to metropoliation Melbourne and Hobart. Future trials should be aware recruitment, eligibility, attendance and adherence rates may differ in other settings.

\section{Conclusion}

A large-scale trial to evaluate the effectiveness of a physiotherapist-guided exercise-therapy and education program for individuals with persistent symptoms at 1year post-ACLR is feasible. All feasibility criteria were met, or reasonable recommendations could be made to achieve the criteria in future trials. Strategies to increase recruitment rate, adherence to exercise and data completion are required. Potential worthwhile treatment effects for knee-related QoL, symptoms and function were observed, indicating a fully-powered RCT may detect a clinically meaningful effect.

\section{Supplementary Information}

The online version contains supplementary material available at https://doi. org/10.1186/s12891-020-03919-6.

Additional file 1. CONSORT Checklist.

Additional file 2. Physiotherapist manual: exercise-therapy and education protocols.

Additional file 3. Lower-limb focussed and trunk-focussed exercisetherapy and education interventions website https://task.trekeducation. org/.

Additional file 4. Feedback from study participants on exercise program content, structure and delivery methods.

Additional file 5. Secondary outcomes additional detail.

\footnotetext{
Abbreviations

ACL: Anterior cruciate ligament; ACLR: Anterior cruciate ligament reconstruction; $A C L-Q o L$ : $A C L$ quality of life survey; $A C L-R S I$ : $A C L$ Return to Sport Index; BMI: Body mass index; Cl: Confidence interval; CERT: Consensus on Exercise Reporting Template; CONSORT: CONsolidated Standards of Reporting Trials; GROC: Global rating of change; KOOS: Knee injury and Osteoarthritis Outcome Score; LSI: Limb symmetry index; MDC: Minimal detectable change; OA: Osteoarthritis; PASS: Patient acceptable symptom state; PROs: Patient reported outcomes; RCT: Randomised controlled trial; SD: Standard deviation; TIDieR: Template for Intervention Description and Replication guidelines; QoL: Quality of life
} 


\section{Acknowledgements}

We thank BodySystem ${ }^{\oplus}$ clinic and Complete Sports Care for providing clinic locations and treating physiotherapists at a reduced cost. We thank Mr. Dirk Van Bavel, Mr. Justin Wong, Mr. Ben Campbell, Mr. Nigel Hartnett, and Mr. Paul Einoder for assisting recruitment into the project.

\section{Authors' contributions}

$B P, A C, K C$ conceived and designed the study, with input from CB and RC. $B P$ recruited participants and collected the pre- and post-intervention data. $\mathrm{BP}, \mathrm{AC}, \mathrm{CB}$ conducted the statistical analysis and interpretation of data, with input from $K C$. BP drafted the manuscript with input from $A C, C B, R C$, and $\mathrm{KC}$. All authors read and approved the final manuscript.

\section{Funding}

Support for this study was provided by the Physiotherapy Research Foundation, Beryl Haines Memorial Grant, and the La Trobe University Social Research Platform. Brooke Patterson, Adam Culvenor and Christian Barton are recipients of National Health and Medical Research Council awards (postgraduate scholarship No. 1114296, Neil Hamilton Fairley Clinical Fellowship No. 1121173 and MRFF Translating Research into Practice No. 11163250, respectively). The sponsors were not involved in the design and conduct of this study, in the analysis and interpretation of the data, and in the preparation, review, or approval of the manuscript.

\section{Availability of data and materials}

The datasets used and/or analysed during the current study are available from the corresponding author on reasonable request.

\section{Ethics approval and consent to participate}

Ethical approval was gained through the La Trobe University Human Ethics Committee (HEC 16-077) and all participants were provided with a written participant information statement and completed written informed consent prior to participating.

\section{Consent for publication}

The images in the instruction booklet in Additional file 2 can be published under the Creative Commons license. Research centre staff pictured provided written consent for their image to be used.

\section{Competing interests}

The authors declare they have no competing interests.

Received: 8 July 2020 Accepted: 26 December 2020

Published online: 11 January 2021

\section{References}

1. American College of Sports Medicine. American College of Sports Medicine position stand. Progression models in resistance training for healthy adults. Med Sci Sports Exerc. 2009:41(3):687-708.

2. Arundale AJH, Capin JJ, Zarzycki R, Smith A, Snyder-Mackler L. Functional and patient-reported outcomes improve over the course of rehabilitation: a secondary analysis of the ACL-SPORTS trial. Sports Health. 2018;10(5):441-52 1941738118779023.

3. Avery KNL, Williamson PR, Gamble C, O'Connell Francischetto E, Metcalfe C, et al. Informing efficient randomised controlled trials: exploration of challenges in developing progression criteria for internal pilot studies. BM. Open. 2017;7(2):e013537.

4. Baltaci G, Yilmaz G, Atay AO. The outcomes of anterior cruciate ligament reconstructed and rehabilitated knees versus healthy knees: a functional comparison. Acta Orthop Traumatol Turc. 2012;46(3):186-95.

5. Bell ML, Whitehead AL, Julious SA. Guidance for using pilot studies to inform the design of intervention trials with continuous outcomes. Clin Epidemiol. 2018;10:153-7.

6. Bennell KL, van Ginckel A, Kean CO, Nelligan RK, French SD, et al. Patient knowledge and beliefs about knee osteoarthritis after anterior cruciate ligament injury and reconstruction. Arthritis Care Res (Hoboken). 2016;68(8): $1180-5$.

7. Bremander $A B, D a h I L L$, Roos EM. Validity and reliability of functional performance tests in meniscectomized patients with or without knee osteoarthritis. Scand J Med Sci Sports. 2007;17(2):120-7.
8. Brewer BW, Van Raalte JL, Cornelius AE, Petitpas AJ, Sklar JH, et al. Psychological factors, rehabilitation adherence, and rehabilitation outcome after anterior cruciate ligament reconstruction. Rehabil Psychol. 2000;45(1): 20-37.

9. Burgi $C R$, Peters $S$, Ardern $C L$, Magill JR, Gomez CD, et al. Which criteria are used to clear patients to return to sport after primary ACL reconstruction? A scoping review. Br J Sports Med. 2019;53(18):1154-61.

10. Collins NJ, Prinsen CA, Christensen R, Bartels EM, Terwee CB, et al. Knee injury and osteoarthritis outcome score (KOOS): systematic review and meta-analysis of measurement properties. Osteoarthr Cartil. 2016;24(8):131729.

11. Coronado RA, Bird ML, Van Hoy EE, Huston LJ, Spindler KP, et al. Do psychosocial interventions improve rehabilitation outcomes after anterior cruciate ligament reconstruction? A systematic review. Clin Rehabil. 2018; 32(3):287-98.

12. Culvenor $\mathrm{A}$, Barton $\mathrm{C}$. It is time to stop wasting time and money debating graft types and surgical approaches for ACL injuries: The secret probably lies in optimising rehabilitation. British J Sports Med. 2017; Available from: https://blogs.bmj.com/bjsm/2017/09/20/. Accessed 7 July 2020.

13. Culvenor AG, Alexander BC, Clark RA, Collins NJ, Ageberg E, et al. Dynamic single-leg postural control is impaired bilaterally following anterior cruciate ligament reconstruction: implications for Reinjury risk. J Orthop Sports Phys Ther. 2016:46(5):357-64.

14. Culvenor AG, Boeth H, Diederichs G, Wirth W, Duda G, et al. Longitudinal bone, muscle and adipose tissue changes in physically active subjects - sex differences during adolescence and maturity. J Musculoskelet Neuronal Interact. 2016;16(3):237-46

15. Culvenor AG, Collins NJ, Guermazi A, Cook JL, Vicenzino B, et al. Early Patellofemoral osteoarthritis features one year after anterior cruciate ligament reconstruction: symptoms and quality of life at three years. Arthritis Care Res (Hoboken). 2016;68(6):784-92.

16. Culvenor AG, Collins NJ, Vicenzino B, Cook JL, Whitehead TS, et al, Predictors and effects of patellofemoral pain following hamstring-tendon ACL reconstruction. J Sci Med Sport. 2016:19(7):518-23.

17. Culvenor AG, Eckstein F, Wirth W, Lohmander LS, Frobell R. Loss of patellofemoral cartilage thickness over 5 years following $\mathrm{ACL}$ injury depends on the initial treatment strategy: results from the KANON trial. Br J Sports Med. 2019:53(18):1168-73.

18. Culvenor AG, Lai CC, Gabbe BJ, Makdissi M, Collins NJ, et al. Patellofemoral $\mathrm{OA}$ is prevalent and associated with worse symptoms and function after hamstring tendon autograft ACL reconstruction. Br J Sports Med. 2014;48(6): 435-9.

19. Culvenor AG, Oiestad BE, Hart HF, Stefanik JJ, Guermazi A, et al. Prevalence of knee osteoarthritis features on magnetic resonance imaging in asymptomatic uninjured adults: a systematic review and meta-analysis. Br J Sports Med. 2019;53(20):1268-78.

20. Culvenor AG, Ruhdorfer A, Juhl C, Eckstein F, Øiestad BE. Knee extensor strength and risk of structural, symptomatic, and functional decline in knee osteoarthritis: a systematic review and meta-analysis. Arthritis Care Res. 2017;69(5):649-58

21. Culvenor AG, Segal NA, Guermazi A, Roemer F, Felson DT, et al. Sex-specific influence of quadriceps weakness on worsening Patellofemoral and Tibiofemoral cartilage damage: a prospective cohort study. Arthritis Care Res (Hoboken). 2019;71(10):1360-5.

22. De Oliveira SD, Pazzinatto MF, Rathleff MS, Holden S, Bell E, et al. Patient education for Patellofemoral pain: a systematic review. J Orthop Sports Phys Ther. 2020;50(7):388-96.

23. Eldridge SM, Chan CL, Campbell MJ, Bond CM, Hopewell S, et al. CONSORT 2010 statement: extension to randomised pilot and feasibility trials. BMJ. 2016:355:5239.

24. Englund M, Roos EM, Lohmander LS. Impact of type of meniscal tear on radiographic and symptomatic knee osteoarthritis: a sixteen-year followup of meniscectomy with matched controls. Arthritis Rheum. 2003;48(8):217887.

25. Ericsson YB, Roos EM, Frobell RB. Lower extremity performance following $\mathrm{ACL}$ rehabilitation in the KANON-trial: impact of reconstruction and predictive value at 2 and 5 years. Br J Sports Med. 2013;47(15):980-5.

26. Filbay SR, Ackerman IN, Dhupelia S, Arden NK, Crossley KM. Quality of life in symptomatic individuals after anterior cruciate ligament reconstruction, with and without radiographic knee osteoarthritis. J Orthop Sports Phys Ther. 2018;48(5):398-408. 
27. Filbay SR, Culvenor AG, Ackerman IN, Russell TG, Crossley KM. Quality of life in anterior cruciate ligament-deficient individuals: a systematic review and meta-analysis. Br J Sports Med. 2015;49(16):1033-41.

28. Furlan AD, Pennick V, Bombardier C, van Tulder M, Editorial Board CBRG 2009 updated method guidelines for systematic reviews in the Cochrane Back review group. Spine (Phila Pa 1976). 2009;34(18):1929-41.

29. Garber CE, Blissmer B, Deschenes MR, Franklin BA, Lamonte MJ, et al. Quantity and quality of exercise for developing and maintaining cardiorespiratory, musculoskeletal, and Neuromotor fitness in apparently healthy adults. Med Sci Sports Exerc. 2011;43(7):1334-59.

30. Goddard K, Roberts C-M, Byron-Daniel J, Woodford L. Psychological factors involved in adherence to sport injury rehabilitation: a systematic review. Int Rev Sport Exerc Psychol. 2020. Advance online publication: https://doi.org/ 10.1080/1750984X.2020.1744179.

31. Green B, Bourne MN, van Dyk N, Pizzari T. Recalibrating the risk of hamstring strain injury (HSI) - a 2020 systematic review and meta-analysis of risk factors for index and recurrent HSI in sport. Br J Sports Med. 2020; 54(18):1081-8. https://doi.org/10.1136/bjsports-2019-100983.

32. Grindem H, Eitzen I, Snyder-Mackler L, Risberg MA. Online registration of monthly sports participation after anterior cruciate ligament injury: a reliability and validity study. Br J Sports Med. 2014;48(9):748-53.

33. Grindem $H$, Snyder-Mackler L, Moksnes $H$, Engebretsen L, Risberg MA. Simple decision rules can reduce reinjury risk by $84 \%$ after $\mathrm{ACL}$ reconstruction: the Delaware-Oslo ACL cohort study. Br J Sports Med. 2016; 50(13):804-8.

34. Gustavsson A, Neeter C, Thomee P, Silbernagel KG, Augustsson J, et al. A test battery for evaluating hop performance in patients with an $A C L$ injury and patients who have undergone ACL reconstruction. Knee Surg Sports Traumatol Arthrosc. 2006;14(8):778-88.

35. Hart HF, Culvenor AG, Guermazi A, Crossley KM. Worse knee confidence, fear of movement, psychological readiness to return-to-sport and pain are associated with worse function after ACL reconstruction. Phys Ther Sport. 2020;41:1-8.

36. Herbert R. Research note: significance testing and hypothesis testing: meaningless, misleading and mostly unnecessary. J Physiother. 2019;65(3): $178-81$.

37. Higgins JPT, Thomas J, Chandler J, Cumpston M, Li T, Page MJ, Welch VA (editors). Cochrane Handbook for Systematic Reviews of Interventions version 6.1 (updated September 2020). Cochrane. 2020. Available from https://www.training.cochrane.org/handbook.

38. Hoffmann TC, Glasziou PP, Boutron I, Milne R, Perera R, et al. Better reporting of interventions: template for intervention description and replication (TIDieR) checklist and guide. BMJ. 2014;348:g1687.

39. Ingelsrud LH, Granan LP, Terwee CB, Engebretsen L, Roos EM. Proportion of patients reporting acceptable symptoms or treatment failure and their associated KOOS values at 6 to 24 months after anterior cruciate ligament reconstruction: a study from the Norwegian knee ligament registry. Am J Sports Med. 2015;43(8):1902-7.

40. Kamper SJ, Maher CG, Mackay G. Global rating of change scales: a review of strengths and weaknesses and considerations for design. J Man Manip Ther. 2009;17(3):163-70.

41. Kemp JL, Coburn SL, Jones DM, Crossley KM. The physiotherapy for Femoroacetabular impingement rehabilitation STudy (physioFIRST): a pilot randomized controlled trial. J Orthop Sports Phys Ther. 2018;48(4):307-15.

42. Kockum B, Heijne Al. Hop performance and leg muscle power in athletes: reliability of a test battery. Phys Ther Sport. 2015;16(3):222-7.

43. Kruse LM, Gray B, Wright RW. Rehabilitation after anterior cruciate ligament reconstruction: a systematic review. J Bone Joint Surg Am. 2012;94(19):1737-48.

44. Kujala UM, Jaakkola LH, Koskinen SK, Taimela S, Hurme M, et al. Scoring of patellofemoral disorders. Arthroscopy. 1993;9(2):159-63.

45. Kvist J, Osterberg A, Gauffin H, Tagesson S, Webster K, et al. Translation and measurement properties of the Swedish version of ACL-return to sports after injury questionnaire. Scand J Med Sci Sports. 2013;23(5):568-75

46. Lafave MR, Hiemstra L, Kerslake S, Heard M, Buchko G. Validity, reliability, and responsiveness of the anterior cruciate ligament quality of life measure: a continuation of its overall validation. Clin J Sport Med. 2017;27(1):57-63.

47. Lancaster GA, Dodd S, Williamson PR. Design and analysis of pilot studies: recommendations for good practice. J Eval Clin Pract. 2004;10(2):307-12.

48. Moher D, Hopewell S, Schulz KF, Montori V, Gotzsche PC, et al. CONSORT 2010 explanation and elaboration: updated guidelines for reporting parallel group randomised trials. Int J Surg. 2012;10(1):28-55.
49. Mohtadi N. Development and validation of the quality of life outcome measure (questionnaire) for chronic anterior cruciate ligament deficiency. Am J Sports Med. 1998;26(3):350-9.

50. Moses B, Orchard J, Orchard J. Systematic review: annual incidence of ACL injury and surgery in various populations. Res Sports Med. 2012;20(3-4):15779.

51. National Health Medical Research Council. National statement on ethical conduct in human research. Canberra: Australian Research Council; 2007.

52. Paradowski PT, Bergman S, Sunden-Lundius A, Lohmander LS, Roos EM. Knee complaints vary with age and gender in the adult population. Population-based reference data for the Knee injury and Osteoarthritis Outcome Score (KOOS). BMC Musculoskelet Disord. 2006;7:38.

53. Patterson B, Culvenor AG, Barton CJ, Guermazi A, Stefanik J, et al. Poor functional performance 1 year after $A C L$ reconstruction increases the risk of early osteoarthritis progression. Br J Sports Med. 2020:54(9):546-53.

54. Patterson BE, Crossley KM, Perraton LG, Kumar AS, King MG, et al. Limb symmetry index on a functional test battery improves between one and five years after anterior cruciate ligament reconstruction, primarily due to worsening contralateral limb function. Phys Ther Sport. 2020;44:67-74.

55. Patterson BE, Culvenor AG, Barton CJ, Guermazi A, Stefanik JJ, et al. Patientreported outcomes one to five years after anterior cruciate ligament reconstruction: the effect of combined injury and associations with osteoarthritis features defined on magnetic resonance imaging. Arthritis Care Res. 2020;72(3):412-22.

56. Patterson BE, Culvenor AG, Barton CJ, Guermazi A, Stefanik JJ, et al. Worsening knee osteoarthritis features on magnetic resonance imaging 1 to 5 years after anterior cruciate ligament reconstruction. Am J Sports Med. 2018:46(12):2873-83.

57. Pinczewski LA, Lyman J, Salmon LJ, Russell VJ, Roe J, et al. A 10-year comparison of ACLR with hamstring tendon and patellar tendon autograft: a controlled, prospective trial. Am J Sports Med. 2007;35(4):564-74.

58. Pizzari T, McBurney H, Taylor NF, Feller JA. Adherence to anterior cruciate ligament rehabilitation: a qualitative analysis. J Sport Rehabil. 2002;11(2):90-102.

59. Pizzari T, Taylor NF, McBurney H, Feller JA. Adherence to rehabilitation after anterior cruciate ligament reconstructive surgery: implications for outcome. J Sport Rehabil. 2005:14(3):201-14.

60. Reid A, Birmingham TB, Stratford PW, Alcock GK, Giffin JR. Hop testing provides a reliable and valid outcome measure during rehabilitation after ACLR. Phys Ther. 2007;87(3):337-49.

61. Reinke EK, Spindler KP, Lorring D, Jones MH, Schmitz L, et al. Hop tests correlate with IKDC and KOOS at minimum of 2 years after primary $\mathrm{ACL}$ reconstruction. Knee Surg Sports Traumatol Arthrosc. 2011;19(11):1806-16.

62. Risberg MA, Oiestad BE, Gunderson R, Aune AK, Engebretsen L, et al. Changes in knee osteoarthritis, symptoms, and function after anterior cruciate ligament reconstruction: a 20-year prospective follow-up study. Am J Sports Med. 2016;44(5):1215-24.

63. Roos EM, Lohmander LS. The knee injury and osteoarthritis outcome score (KOOS): from joint injury to osteoarthritis. Health Qual Life Outcomes. 2003; 1:64.

64. Slade SC, Dionne CE, Underwood M, Buchbinder R. Consensus on exercise reporting template (CERT): explanation and elaboration statement. $\mathrm{Br}$ J Sports Med. 2016;50(23):1428-37.

65. Spindler KP, Huston LJ, Chagin KM, Kattan MW, Reinke EK, et al. Ten-year outcomes and risk factors after anterior cruciate ligament reconstruction: a MOON longitudinal prospective cohort study. Am J Sports Med. 2018;46(4): 815-25.

66. Sritharan $P$, Schache $A G$, Culvenor AG, Perraton LG, Bryant AL, et al. Between-limb differences in patellofemoral joint forces during running 12 to 24 months after unilateral ACL reconstruction. Am J Sports Med. 2020; 48(7):1711-9.

67. Tan JM, Menz HB, Crossley KM, Munteanu SE, Hart HF, et al. The efficacy of foot orthoses in individuals with patellofemoral osteoarthritis: a randomised feasibility trial. Pilot Feasibility Studies. 2019;5(1):90

68. Thorstensson CA, Petersson IF, Jacobsson LT, Boegard TL, Roos EM. Reduced functional performance in the lower extremity predicted radiographic knee osteoarthritis five years later. Ann Rheum Dis. 2004;63(4):402-7.

69. Toigo M, Boutellier U. New fundamental resistance exercise determinants of molecular and cellular muscle adaptations. Eur J Appl Physiol. 2006;97(6): 643-63.

70. Truong LK, Mosewich AD, Holt CJ, Le CY, Miciak M, et al. Psychological, social and contextual factors across recovery stages following a sport- 
related knee injury: a scoping review. Br J Sports Med. 2020;54(19):1149-56. https://doi.org/10.1136/bjsports-2019-101206.

71. Tubach F, Dougados M, Falissard B, Baron G, Logeart I, et al. Feeling good rather than feeling better matters more to patients. Arthritis Rheum. 2006; 55(4):526-30.

72. van Melick N, van Cingel RE, Brooijmans F, Neeter C, van Tienen T, et al. Evidence-based clinical practice update: practice guidelines for anterior cruciate ligament rehabilitation based on a systematic review and multidisciplinary consensus. Br J Sports Med. 2016;50(24):1506-15.

73. Walker A, Hing W, Lorimer A. The Influence, Barriers to and Facilitators of Anterior Cruciate Ligament Rehabilitation Adherence and Participation: a Scoping Review. Sports Med Open. 2020;6(1):32.

74. Webster KE, Feller JA, Lambros C. Development and preliminary validation of a scale to measure the psychological impact of returning to sport following anterior cruciate ligament reconstruction surgery. Phys Ther Sport. 2008;9(1):9-15

75. Webster KE, Nagelli CV, Hewett TE, Feller JA. Factors associated with psychological readiness to return to sport after anterior cruciate ligament reconstruction surgery. Am J Sports Med. 2018;46(7):1545-50.

76. Whittaker JL, Roos EM. A pragmatic approach to prevent post-traumatic osteoarthritis after sport or exercise-related joint injury. Best Pract Res Clin Rheumatol. 2019:33(1):158-71.

77. Wilson $\mathrm{K}$, Brookfield D. Effect of goal setting on motivation and adherence in a six-week exercise program. Int J Sport Exerc Psychol. 2009;7(1):89-100.

78. Wright RW, Gill CS, Chen L, Brophy RH, Matava MJ, et al. Outcome of revision anterior cruciate ligament reconstruction: a systematic review. J Bone Joint Surg Am. 2012;94(6):531-6.

79. Xergia SA, Pappas E, Zampeli F, Georgiou S, Georgoulis AD. Asymmetries in functional hop tests, lower extremity kinematics, and isokinetic strength persist 6 to 9 months following anterior cruciate ligament reconstruction, J Orthop Sports Phys Ther. 2013;43(3):154-62.

\section{Publisher's Note}

Springer Nature remains neutral with regard to jurisdictional claims in published maps and institutional affiliations.

Ready to submit your research? Choose BMC and benefit from:

- fast, convenient online submission

- thorough peer review by experienced researchers in your field

- rapid publication on acceptance

- support for research data, including large and complex data types

- gold Open Access which fosters wider collaboration and increased citations

- maximum visibility for your research: over $100 \mathrm{M}$ website views per year

At $\mathrm{BMC}$, research is always in progress.

Learn more biomedcentral.com/submissions 